\title{
Haplo-insufficiency of both BubR1 and SGO1 accelerates cellular senescence
}

\author{
Sung-Hyun Park', Steve Xie ${ }^{2}$, Chinthalapally V. Rao ${ }^{3}$ and Wei Dai ${ }^{*}$
}

\begin{abstract}
Background: Spindle assembly checkpoint components BubR1 and Sgo1 play a key role in the maintenance of chromosomal instability during cell division. These proteins function to block the anaphase entry until all condensed chromosomes have been attached by the microtubules emanating from both spindle poles. Haplo-insufficiency of either BUbR1 or SGO1 results in enhanced chromosomal instability and tumor development in the intestine. Recent studies show that spindle checkpoint proteins also have a role in slowing down the ageing process. Therefore, we want to study whether haplo-insufficiency of both BubR1 and SGO1 accelerates cellular senescence in mice.

Methods: We took advantage of the availability of BubR1 and SGO1 knockout mice and generated primary murine embryonic fibroblasts (MEFs) with mutations in either BubR1, SGO1, or both and analyzed cellular senescence of the MEFs of various genetic backgrounds.

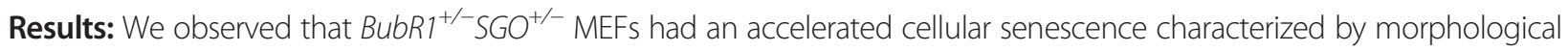
changes and expressed senescence-associated $\beta$-galactosidase. In addition, compared with wild-type MEFs or MEFs with a single gene deficiency, BubR1 ${ }^{+/-} \mathrm{SGO} 1^{+/-}$MEFs expressed enhanced levels of p21 but not p16.

Conclusions: Taken together, our observations suggest that combined deficiency of BubR1 and Sgo1 accelerates cellular senescence.
\end{abstract}

Keywords: BubR1, SGO1, Knockout mice, MEFs, Senescence

\section{Background}

Senescent cells are characterized as having gradually lost the ability to divide. Senescence is the main risk factor for age-related disorders including diabetes, atherosclerosis, hypertension, osteoarthritis, and Alzheimer's disease and Parkinson's disease [1]. There are two types of senescence including replicative senescence and premature senescence. The replicative senescence is driven largely by telomere shortening [2], whereas the premature senescence is characterized by permanent cell cycle arrest without telomere deterioration [3]. Senescence can be induced by a variety of stressors including UV, oxidative stress, genomic instability, free radical accumulation, and inflammatory cytokines. Senescent cells are characterized by an increased cell size and expression of senescence-associated $\beta$-galactosidase $(\mathrm{SA}-\beta-\mathrm{Gal})$. In

\footnotetext{
* Correspondence: wei.dai@nyumc.org

'Departments of Environmental Medicine, Biochemistry \& Molecular Pharmacology, New York University Langone Medical Center, 57 Old Forge Road, Tuxedo, New York 10987, USA

Full list of author information is available at the end of the article
}

addition, senescent cells undergo permanent cell cycle arrest, which is accompanied by expressing enhanced levels of negative regulators for cell division including p21, p16, and PTEN.

Spindle checkpoint proteins play a key role in the maintenance of chromosomal instability during cell division [4]. BubR1 is one of spindle checkpoint proteins which functions to block the anaphase entry until all chromosomes have been attached by the microtubules emanating from both spindle poles. Homozygous deletion of BubR1 results in embryonic lethality [5]. BubR1 haplo-insufficiency $\left(B u b R 1^{+/-}\right)$causes enhanced chromosomal instability and tumorigenesis [6]. Extensive research in the past has shown that BubR1-deficient mice also exhibit age-related phenotypes including short lifespan, cachectic dwarfism, lordokyphosis, cataracts, and impaired wound healing $[7,8]$. In contrast, increased expression of BubR1 extends healthy lifespan in a mouse model [9]. Therefore, spindle checkpoint protein BubR1 plays an important role in suppressing ageing. 
Shugoshin 1 (Sgo1) functions to maintain sister chromatid cohesion, preventing premature cleavage of the cohesin complex by separase [10, 11]. Cells with Sgo1 depletion experience difficulty in completing chromosome congression, leading to accumulation of clusters of chromosomes that surround the spindle poles [12-14]. In the absence of Sgo1, the metaphase plate collapses prior to completion of congression of all chromosome pairs [12]. Missegregation of sister chromatids induced by Sgo1 depletion appears to be responsible for dramatic mitotic arrest [15]. A splice variant of Sgo1 is also involved in the maintenance of centriole cohesion $[15,16]$. Similar to many spindle checkpoint proteins, haploinsufficiency of SGO1 also predisposes mice to the development of malignancies [10].

Given the availability of BubR1 and SGO1 heterozygous mice, we generated primary murine embryonic fibroblasts (MEFs) with mutations in either BuBR1, $S G O 1$, or both. We studied whether deficiency in the checkpoint gene(s) had an impact on cellular senescence. We observed that $\mathrm{BubR}^{+/-}$and $\mathrm{SGO}^{+/-} \mathrm{MEFs}$ had an accelerated cellular senescence characterized by the morphological changes and expression of SA- $\beta$-Gal. Moreover, double mutant MEFs $\left(\mathrm{BubR}^{+/-} \mathrm{SGO1}^{+/-}\right)$exhibited the highest rate of cellular senescence, which was accompanied by early appearance of morphological changes and expression of SA- $\beta-$ Gal. Compared with wild-type MEFs or MEFs with a single gene deficiency,

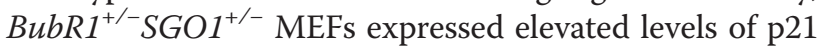
but not p16.

\section{Methods}

\section{Mouse colonies and MEFs}

Transgenic knockout mice $\left(B u b R 1^{+/-}\right.$mice and $S G O 1^{+/-}$ mice) were maintained at the animal facility of $\mathrm{New}$ York University Langone Medical Center. The maintenance was fully compliant with the general guidelines outlined by the United States Department of Agriculture and the American Association of Laboratory Animal Care under the supervision and specific approval of the Institutional Animal Care and Use Committees. BubR1 ${ }^{+/} \mathrm{SGOI}^{+/-}$compound mutant mice were obtained by crossing $\mathrm{BubR}^{+/-}$mice with $\mathrm{SGO1}^{+/-}$mice. Primary MEFs of various genotypes were obtained from embryos (embryonic day 14.5) of mice of $\mathrm{BubR}^{+/-}$and $\mathrm{SGO1}^{+/-}$ crossing. MEF cells were cultured at $37{ }^{\circ} \mathrm{C}$ under $5 \%$ $\mathrm{CO}_{2}$ in $100-\mathrm{mm}$ plates containing Dulbecco minimum essential medium (DMEM) supplemented with $10 \%$ fetal bovine serum (FBS) and antibiotics $(100 \mu \mathrm{g} / \mathrm{ml}$ of penicillin and $50 \mu \mathrm{g} / \mathrm{ml}$ of streptomycin sulfate; Invitrogen). Serial cultures were carried out in which cells were trypsinized and replated at a density of $10^{6}$ cells $/ 100-\mathrm{mm}$ dish every 7 days.

\section{Genotyping}

Genotyping was carried out as previously described $[5,10]$. Briefly, genomic DNA was purified from mouse tail or MEFs. The PCR for detecting BubR1 genotype was carried out with PCR primers as follows: the forward primer A of 5 'GGG AGG ATC GAG GAG GTC G3', the forward primer longterminal repeat 2 (LTR2) of 5'AAA TGG CGT TAC TTA AGC TAG CTT GC3', and the reverse primer B of 5'CTG TTC GCC TTC AGT GCT CAA AAT GGT AGT CG3'. The reaction conditions included an initial denaturation at $94{ }^{\circ} \mathrm{C}$ for $5 \mathrm{~min}$ followed by 35 cycles of $94{ }^{\circ} \mathrm{C}$ for $30 \mathrm{~s}, 58{ }^{\circ} \mathrm{C}$ for $30 \mathrm{~s}$, and $72{ }^{\circ} \mathrm{C}$ for $1 \mathrm{~min}$. The final extension of the reactions was set at $72{ }^{\circ} \mathrm{C}$ for $7 \mathrm{~min}$. The PCR for SGO1 genotyping was carried out with primers as follows: the forward primer of 5'GAA AAG TAA GTC TGC TTA TGG CTC A3', the reverse primer of 5'CAG GTG TTG TAG AAT AAT CCA AGC3', and the reverse primer long-terminal repeat (LTR) of 5'ATA AAC CCT CTT GCA GTT GCA TC3'. The reaction conditions included an initial denaturation at $94{ }^{\circ} \mathrm{C}$ for $3 \mathrm{~min}$ followed by 35 cycles of $94{ }^{\circ} \mathrm{C}$ for $45 \mathrm{~s}, 57{ }^{\circ} \mathrm{C}$ for $45 \mathrm{~s}$, and $72{ }^{\circ} \mathrm{C}$ for $1 \mathrm{~min}$. The final extension was set at $72{ }^{\circ} \mathrm{C}$ for $10 \mathrm{~min}$. PCR products were analyzed by electrophoresis on $2 \%$ agarose gels and detected by ethidium bromide staining.

\section{Senescence-associated $\beta$-galactosidase staining}

MEF cells were seeded at a density of $3 \times 10^{4}$ cells (at passages 3 and 4 ), $4 \times 10^{4}$ cells (at passage 5 ), $5 \times 10^{4}$ cells (at passage 6), $6 \times 10^{4}$ cells (at passage 7), and $7 \times$ $10^{4}$ cells (at passage 8 ) per well, respectively, on 12-well plates. One day after seeding, MEFs were stained for SA$\beta$-Gal activity according to the manufacturer's protocol (Cell Signaling Technology). The percentage of senescent cells was the total number of senescent cells divided by the total number of cells counted under a light microscope.

\section{Western blot analysis}

Western blot analysis was carried out as previously described $[17,18]$. Total MEF cell lysates were prepared in the RIPA lysis buffer (50 mM Tris- $\mathrm{HCl}$ (pH 8.0), $150 \mathrm{mM}$ $\mathrm{NaCl}, 1 \% \mathrm{NP}-40,0.1 \%$ SDS, and $0.5 \%$ sodium deoxycholate) supplemented with a mixture of protease and phosphatase inhibitors. Protein concentrations were measured using the bicinchoninic acid protein assay (Pierce ${ }^{\mathrm{Tu}}$ BCA Protein Assay Kit, ThermoFisher Scientific). Equal amounts of protein lysates from various samples were used for SDSPAGE analysis followed by immunoblotting. Luminata Forte Western HRP substrate (Millipore) was used for chemiluminescent detection. 


\section{Statistical analysis}

Statistical analysis and graphs were generated using the Prism 6 (Graphpad). Data were analyzed for significant differences using the two-tailed, independent $t$ test with Welch's correction for un-equal variances, or with the Mann-Whitney $U$ test. A $p$ value $<0.05$ was considered statistically significant.

\section{Results and discussion}

It is well documented that deficiency in spindle checkpoint control results in chromosomal instability, aneuploidy, and tumorigenesis [19-21]. Recent studies have revealed that aneuploidy due to a weakened spindle checkpoint accelerates ageing [7]. For example, mice carrying hypomorphic BubR1 alleles develop a series of ageing-related pathologies that include shortened life span, growth retardation, fat loss, and impaired wound healing [7]. Given that SGO1 also plays a role in the maintenance of chromosomal stability during cell division and that it remains unclear whether SGO1-deficiency also contributes to the accelerated ageing process, we first obtained primary MEFs that were either haploinsufficiency of BubR1,SGO1, or both. Morphological examination revealed that SGO1-deficient MEFs appeared to be similar to that of BubR1-deficient ones, which grew at a slightly faster rate than the wild-type MEFs (data not shown). However, compound mutant MEFs $\left(B u b R 1^{+/-} S G O 1^{+/-}\right)$grew at a much slower rate, and a small fraction of these cells exhibited morphologies of senescent cells at early passages (e.g., P4) (Fig. 1).
Many MEFs became large, flat, and/or multinucleated. With each passage, these morphologically distinct cells significantly increased in $\mathrm{BubR}^{+/-} \mathrm{SGO1}^{+/-}$MEFs compared with wild-type MEFs. By passage 8 , a high fraction of $\mathrm{BubR}^{+/-} \mathrm{SGO1}^{+/-}$displayed senescent cell morphologies. These senescent-like cells were also present in BubR1- and SGO1-deficient cells at late passage (passage 8).

Given that lysosomal SA- $\beta-$ Gal is a reliable and widely used biomarker for senescent cells, we stained MEFs of various genotypes for SA- $\beta$-Gal expression. We observed that a significant number of $\mathrm{BubR}^{1^{+-}} \mathrm{SGO1}^{+/-} \mathrm{MEFs}$ at very early passages (e.g., passage 3 ) already expressed SA- $\beta$-Gal where few, if any, wild-type MEFs expressed this enzyme (Fig. 2). Some MEFs with a single gene deficiency $\left(\mathrm{BubR}^{+/-}\right.$or $\left.S G O 1^{+/-}\right)$also expressed SA- $\beta-\mathrm{Gal}$, indicative of their senescent status. However, MEFs with compound mutations displayed a much higher tendency toward senescence. At passage 6, a significant number of $B u b R 1^{+/-}$or $S G O 1^{+/-}$MEFs also expressed SA- $\beta$-Gal

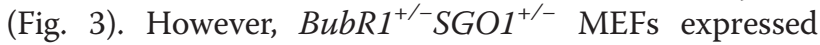
SA- $\beta$-Gal at a higher level, evidenced by the stronger signals of SA- $\beta$-Gal than MEFs with a single gene deficiency.

We then systematically analyzed SA- $\beta$-Gal expression in MEFs of various genotypes at different passages. We observed that a small fraction (around $3 \%$ ) of wild-type MEFs started to express SA- $\beta$-Gal at passage 4 , and there was gradual increase in the percentage of SA- $\beta$-Gal-posi-

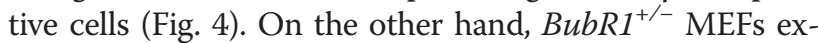
hibited a significant increase of SA- $\beta$-Gal-positive cells

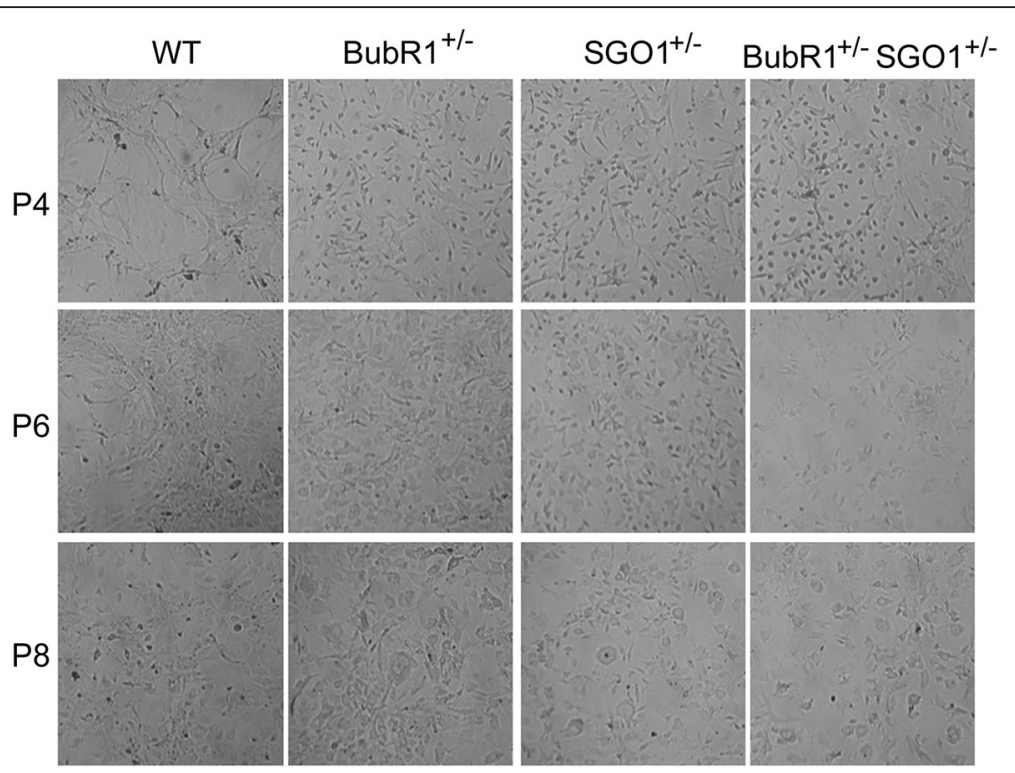

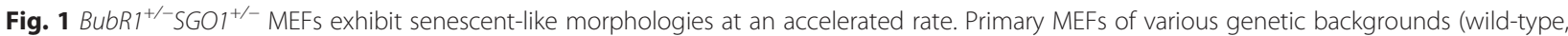

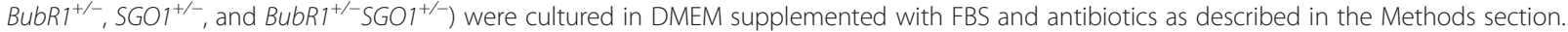
Serial cultures were carried out at a density of $10^{6}$ cells/100-mm dish every 7 days. At each passage, images of MEFs were recorded with a light microscope. Images of MEFs of wild-type $(W T), B u b R 1^{+/-}, \mathrm{SGO}^{+/-}$, and $B \mathrm{BuR1^{+/- }} \mathrm{SGO}^{+/-}$at passages 4, 6, and 8 are shown 
WT
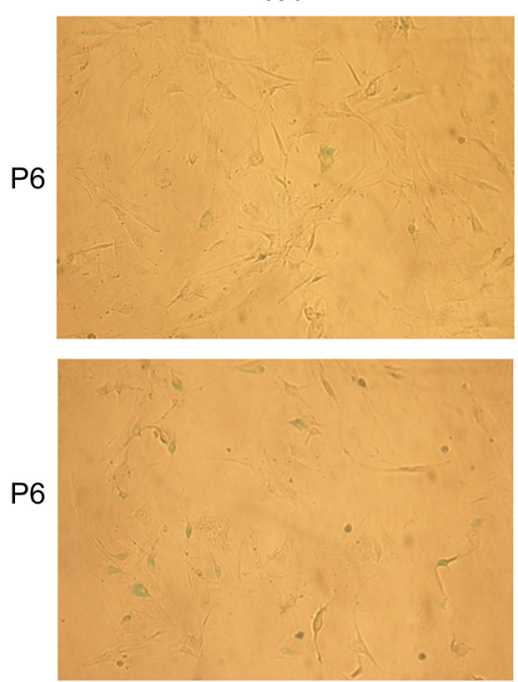

$\mathrm{SGO}^{+/-}$
BubR1 $^{+/-}$
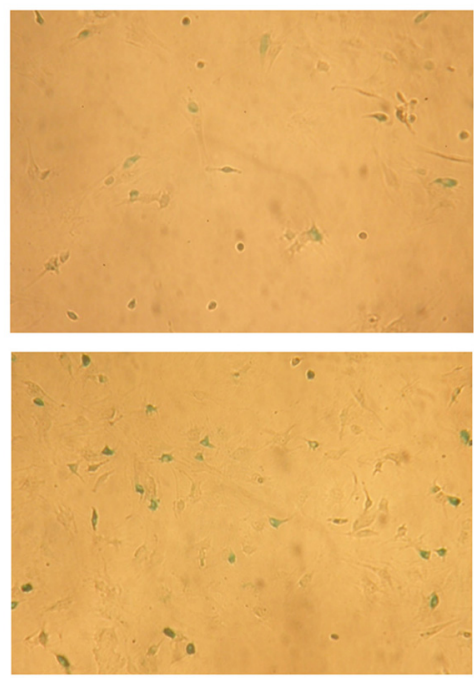

BubR $^{+/-}$SGO1 ${ }^{+/-}$

Fig. $2 \mathrm{BubR}^{+/-}$or $\mathrm{SGO} 1^{+/-}$deficiency leads to enhanced expression of $\beta$-galactosidase. Primary MEFs of various genetic backgrounds (wild-type,

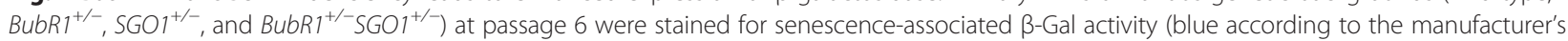
protocol. $\beta$-Gal-positive cells were stained with blue

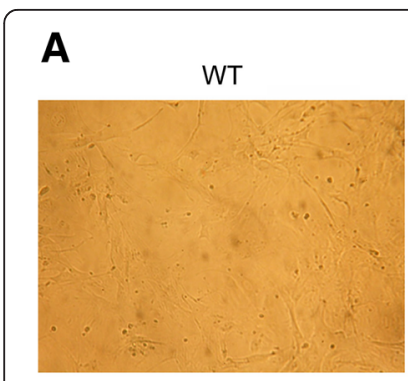

P3

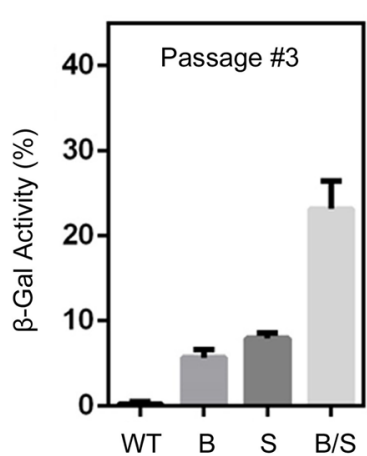

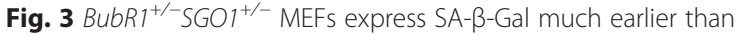
wild-type $B u b R 1^{+/-}$or $S G O 1^{+/-}$MEFs. a Primary MEFs of various genetic backgrounds (wild-type and $\mathrm{BubR}^{+/-} \mathrm{SGO}^{+/-}$) at passage 3 were stained for senescence-associated $\beta$-Gal activity. $\mathbf{b}$ Primary MEFs of various genetic

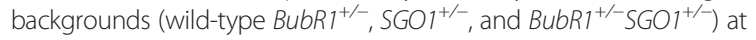
passage 3 were stained for senescence-associated $\beta$-Gal activity. $\beta$-Galpositive cells were recorded. Summarized data from three independent experiments are shown

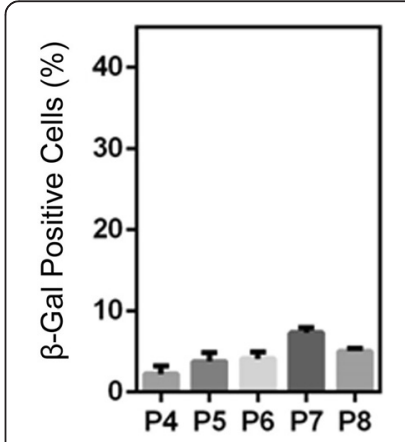

WT

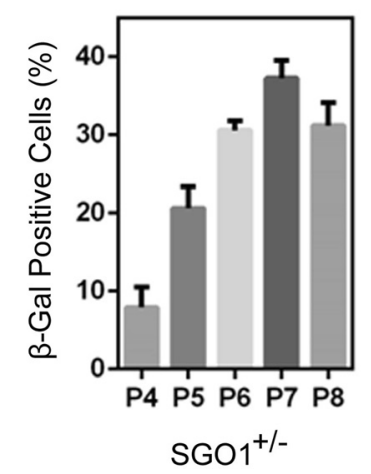

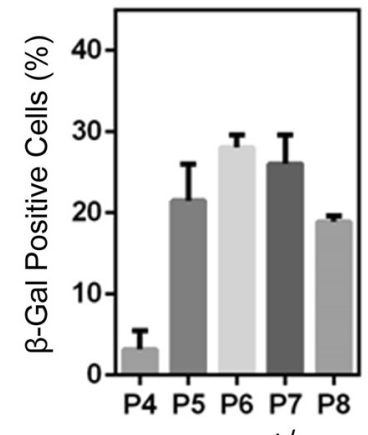

BubR1 $^{+/-}$

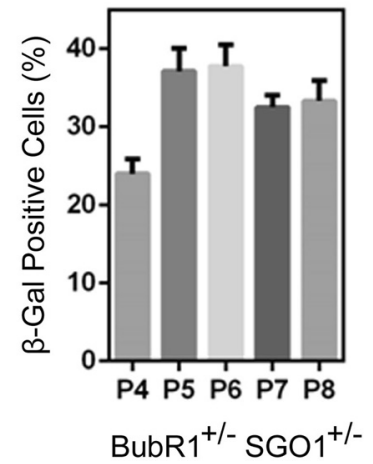

Fig. 4 BubR $1^{+/-} \mathrm{SGO}^{+/-}$compound mutations accelerate senescence. Primary MEFs of various genetic backgrounds (wild-type,

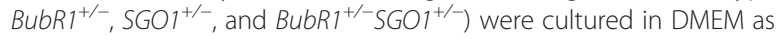
described in the Methods section. MEFs of different passages were stained for SA- $\beta$-Gal. Percents of SA- $\beta$-Gal cells were recorded. Data summarized from three independent experiments are presented 
around passage 5, reaching the peak level at passage 6 . Slightly different from $\mathrm{BubR}^{+/-}$MEFs, $\mathrm{SGO1}^{+/-} \mathrm{MEFs}$ showed a significant increase in the percentage of SA- $\beta$ Gal-positive cells at passage 4, reaching the peak level at passage 7 . The percentage of SA- $\beta$-Gal-positive cells in $S G O 1^{+/-}$MEFs was higher than that in $\mathrm{BubR}^{+/-}$MEFs. Moreover, in addition to early (passage 3 ) display of cellular senescence, $B u b R 1^{+/-} S G O 1^{+/-}$MEFs also had a higher percentage of cells positive for SA- $\beta-$ Gal at passages 4 and 5 . It appears that the highest percentage of SA- $\beta-$-Gal-positive cells in all genotypes was around $37 \%$. Thus, there is a synergy of premature ageing between reduced expression of BubR1 and SGO1.

To further confirm accelerated ageing due to the reduced expression of spindle checkpoint genes, we analyzed several molecular markers including p21 and p16 that are frequently linked to cellular senescence. At pas-

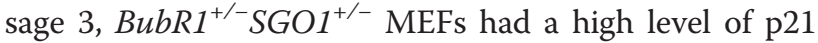
expression compared with that of $B u b R 1^{+/-}$or $S G O 1^{+/-}$ MEFs (Fig. 5). Expression of p21 further increased with additional passages, which was roughly correlated with percentages of SA- $\beta$-Gal-positive cells. On the other hand, there was little difference of p16 expression among early passages until passages 7 and 8 in BubR1 ${ }^{+/} \mathrm{SGO1}^{+/-} \mathrm{MEFs}$. Moreover, there was no significant difference of p16 expression between BubR1 (or SGO1)deficient and BubR1 (or SGO1)-competent MEFs. These observations suggest that p16 expression may not be responsible for the observed cellular senescence in MEFs that are haplo-insufficiency of BubR1, SGO1, or both.

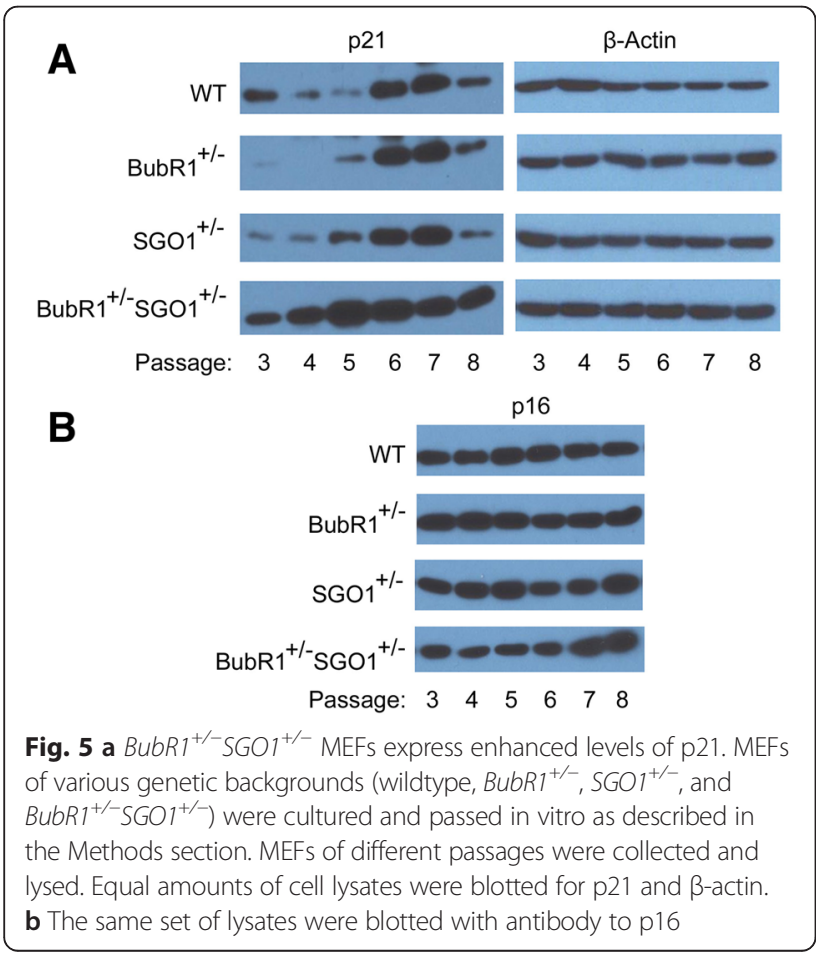

In this study, we systematically analyzed cellular senescence in cells deficient in BubR1 and/or Sgo1. It is known that reduced expression of BubR1 induces premature ageing which is associated with enhanced cellular senescence [7]. We have shown for the first time that haplo-insufficiency of $S G O 1$ also causes increased senescence. Sgo1 appears to play a more important role in suppressing ageing as haplo-insufficiency of SGO1 induces cellular senescence earlier and to a greater extent than haplo-insufficiency of BubR1. At the molecular level, BubR1 and Sgo1 function differently in suppressing premature separation of sister chromatids [20]. BubR1 is an integral component of the spindle checkpoint by suppressing the activity of $\mathrm{APC} / \mathrm{C}[22,23]$, whereas Sgol is physically associated with cohesin, preventing its premature cleavage by separase. Therefore, it is reasonable to predict that there is a synergy between BubR1 and Sgo1 in suppressing premature sister chromatid separation and chromosomal instability in cell division. Consistent with this prediction, we have observed that reduced expression of both BubR1 and Sgo1 results in accelerated cellular senescence.

We have shown that enhanced senescence is correlated with elevated expression of p21 but not p16. As it is well known that cellular senescence is associated with permanent cell cycle arrest, it is logical that expression of CDK inhibitors such as p21 is significantly elevated. Although we did not observe that p16 expression is high in MEFs that are undergoing senescence, we cannot exclude the possibility that expression of other inhibitors of the INK family including p15, p17, or p19 is elevated. Although they all function to block cell cycle progression, p21 and p16 act differently in the negative regulation of CDKs. As a member of the INK family, p16 solely acts as an inhibitor that blocks G1 CDKs (CDK4/ 6). On the other hand, p21 is capable of inhibiting CDKs of several cell cycle transitions including $\mathrm{S}$ and G2/M. Given that BubR1 and Sgo1 have primary functions in mitosis, it is logical to speculate that impaired functions of these checkpoint components can trigger cell cycle arrest and senescence by activating p 21 expression.

\section{Conclusions}

This study is consistent with the notion that chromosomal instability due to reduced expression of spindle assembly checkpoint components contributes to ageing processes. The combined deficiency of BubR1 and $S G O 1$ accelerates cellular senescence which is manifested as morphological changes and enhanced expression of SA- $\beta-$ Gal.

Competing interests

The authors declare that they have no competing interests. 


\section{Authors' contributions}

SP: Designed and executed experiments. Also involved in data interpretation and manuscript writting. SX: Helped with data interpretation and discussion. CVR: Provided key reagents. Data discussion. WD: Designed experiments. Data interpretations and manuscript writting. All authors read and approved the final manuscript.

\section{Acknowledgements}

We thank the lab members for the valuable discussions during the course of the study. This work was supported in part by the US Public Service Awards to WD (CA090658 \& CA150512) and NIEHS Center grant (ES000260).

\section{Author details}

${ }^{1}$ Departments of Environmental Medicine, Biochemistry \& Molecular Pharmacology, New York University Langone Medical Center, 57 Old Forge Road, Tuxedo, New York 10987, USA. ${ }^{2}$ Institute of Pathology, Kings County Hospital Center, Brooklyn, New York, USA. ${ }^{3}$ Center for Cancer Prevention and Drug Development Program, Department of Medicine, Hem/Onc Section, University of Oklahoma Health Sciences Center, Oklahoma, USA.

Received: 15 October 2015 Accepted: 28 January 2016

Published online: 04 February 2016

\section{References}

1. Naylor RM, Baker DJ, van Deursen JM. Senescent cells: a novel therapeutic target for aging and age-related diseases. Clin Pharmacol Ther. 2013;93(1):105-16.

2. Wang $L$, Xiao $H$, Zhang $X$, Wang $C$, Huang $H$. The role of telomeres and telomerase in hematologic malignancies and hematopoietic stem cell transplantation. J Hematol Oncol. 2014;7:61.

3. Shay JW, Wright WE. Hayflick, his limit, and cellular ageing. Nat Rev Mol Cell Biol. 2000;1(1):72-6.

4. Noll JE, Vandyke K, Hewett DR, Mrozik KM, Bala RJ, Williams SA. PTTG1 expression is associated with hyperproliferative disease and poor prognosis in multiple myeloma. J Hematol Oncol. 2015;8(1):106.

5. Wang Q, Liu T, Fang Y, Xie S, Huang X, Mahmood R. BUBR1 deficiency results in abnormal megakaryopoiesis. Blood. 2004;103(4):1278-85.

6. Dai W, Wang Q, Liu T, Swamy M, Fang Y, Xie S. Slippage of mitotic arrest and enhanced tumor development in mice with BubR1 haploinsufficiency. Cancer Res. 2004:64(2):440-5.

7. Baker DJ, Jeganathan KB, Cameron JD, Thompson M, Juneja S, Kopecka A. BubR1 insufficiency causes early onset of aging-associated phenotypes and infertility in mice. Nat Genet. 2004;36(7):744-9.

8. Hartman TK, Wengenack TM, Poduslo JF, van Deursen JM. Mutant mice with small amounts of BubR1 display accelerated age-related gliosis. Neurobiol Aging. 2007;28(6):921-7.

9. Baker DJ, Dawlaty MM, Wijshake T, Jeganathan KB, Malureanu L, van Ree JH. Increased expression of BubR1 protects against aneuploidy and cancer and extends healthy lifespan. Nat Cell Biol. 2013;15(1):96-102.

10. Yamada HY, Yao Y, Wang X, Zhang Y, Huang Y, Dai W. Haploinsufficiency of SGO1 results in deregulated centrosome dynamics, enhanced chromosomal instability and colon tumorigenesis. Cell Cycle. 2012;11(3):479-88.

11. Yao Y, Dai W. Shugoshins function as a guardian for chromosomal stability in nuclear division. Cell Cycle. 2012;11(14):2631-42.

12. Kitajima TS, Kawashima SA, Watanabe Y. The conserved kinetochore protein shugoshin protects centromeric cohesion during meiosis. Nature. 2004; 427(6974):510-7.

13. Tang Z, Sun Y, Harley SE, Zou H, Yu H. Human Bub1 protects centromeric sister-chromatid cohesion through Shugoshin during mitosis. Proc Natl Acad Sci U S A. 2004;101(52):18012-7.

14. Kitajima TS, Hauf S, Ohsugi M, Yamamoto T, Watanabe Y. Human Bub1 defines the persistent cohesion site along the mitotic chromosome by affecting Shugoshin localization. Current biology : CB. 2005;15(4):353-9.

15. Wang $X$, Yang $Y$, Dai W. Differential subcellular localizations of two human Sgo1 isoforms: implications in regulation of sister chromatid cohesion and microtubule dynamics. Cell Cycle. 2006:5(6):635-40.

16. Wang $X$, Yang Y, Duan Q, Jiang N, Huang Y, Darzynkiewicz Z. sSgo1, a major splice variant of Sgo1, functions in centriole cohesion where it is regulated by PIk1. Dev Cell. 2008;14(3):331-41.
17. Chao MW, Lai MJ, Liou JP, Chang YL, Wang JC, Pan SL. The synergic effect of vincristine and vorinostat in leukemia in vitro and in vivo. J Hematol Oncol. 2015;8:82.

18. lacobucci I, Di Rora AG, Falzacappa MV, Agostinelli C, Derenzini E, Ferrari A. In vitro and in vivo single-agent efficacy of checkpoint kinase inhibition in acute lymphoblastic leukemia. J Hematol Oncol. 2015;8(1):125.

19. Holland AJ, Cleveland DW. Boveri revisited: chromosomal instability, aneuploidy and tumorigenesis. Nat Rev Mol Cell Biol. 2009;10(7):478-87.

20. Dai W. Suppression of genomic instabilities caused by chromosome missegregation: a perspective from studying BubR1 and Sgo1. J Formos Med Assoc. 2009:108(12):904-11.

21. Schiaffino-Ortega S, Balinas C, Cuadros M, Medina PP. SWI/SNF proteins as targets in cancer therapy. J Hematol Oncol. 2014;7:81.

22. Wu H, Lan Z, Li W, Wu S, Weinstein J, Sakamoto KM. p55CDC/hCDC20 is associated with BUBR1 and may be a downstream target of the spindle checkpoint kinase. Oncogene. 2000;19(40):4557-62.

23. Choi $\mathrm{E}$, Choe H, Min J, Choi JY, Kim J, Lee H. BubR1 acetylation at prometaphase is required for modulating APC/C activity and timing of mitosis. EMBO J. 2009;28(14):2077-89.

\section{Submit your next manuscript to BioMed Central and we will help you at every step:}

- We accept pre-submission inquiries

- Our selector tool helps you to find the most relevant journal

- We provide round the clock customer support

- Convenient online submission

- Thorough peer review

- Inclusion in PubMed and all major indexing services

- Maximum visibility for your research

Submit your manuscript at www.biomedcentral.com/submit 